\title{
Comparison between femtosecond laser and fusion-arc inscribed long period gratings in photonic crystal fibre
}

\author{
T. Allsop ${ }^{1 *}$, K. Kalli ${ }^{2 *}$, K. Zhou ${ }^{1}$, G. Smith ${ }^{1}$, M. Komodromos ${ }^{3}$, K. Sugden ${ }^{1}$, M. Dubov ${ }^{1}$, D.J. Webb ${ }^{1}$, \\ I. Bennion ${ }^{1}$ \\ ${ }^{1}$ Photonics Research Group, Aston University, Aston Triangle, Birmingham, B4 7ET, UK \\ ${ }^{2}$ Cyprus University of Technology, Department of Electrical Engineering and Information \\ Technology, 31 Archbishop Kyprianos, Lemessos 3036, Cyprus \\ ${ }^{3}$ Frederick Institute of Technology, 7 Filokyprou, Nicosia, Cyprus
}

\begin{abstract}
The use of high intensity femtosecond laser sources for inscribing fibre gratings has attained significant interest. The principal advantage of high-energy pulses is their ability for grating inscription in any material type without preprocessing or special core doping. In the field of fibre optical sensing LPGs written in photonic crystal fibre have a distinct advantage of low temperature sensitivity over gratings written in conventional fibre and thus minimal temperature cross-sensitivity. Previous studies have indicated that LPGs written by a point-by-point inscription scheme using a low repetition femtosecond laser exhibit post-fabrication evolution leading to temporal instabilities at room temperatures with respect to spectral location, strength and birefringence of the attenuation bands. These spectral instabilities of LPGs are studied in photonic crystal fibres (endlessly single mode microstructure fibre) to moderately high temperatures $100^{\circ} \mathrm{C}$ to $200^{\circ} \mathrm{C}$ and their performance compared to fusion-arc fabricated LPG. Initial results suggest that the fusion-arc fabricated LPG demonstrate less spectral instability for a given constant and moderate temperature, and are similar to the results obtained when inscribed in a standard single mode fibre.
\end{abstract}

Keywords: Photonic Crystal Fibre, Annealing, Femtosecond laser, Fusion-Arc, Long Period Grating

\section{INTRODUCTION}

Long period gratings are currently the subject of considerable research interest due to their potential applications as filters and as sensing devices, responsive to strain, temperature, bending and refractive index [1]. Compared to the more mature fibre Bragg grating sensors, LPGs have more complex spectra, usually with broader spectral features. On the other hand they are intrinsically sensitive to bending and refractive index, which FBGs are not. Perhaps more importantly, the fibre design and choice of grating period can have a considerable influence over the sensitivity to the various parameters, for example allowing the creation of a bend sensor with minimal temperature cross-sensitivity. Along with the increasing design flexibility of optical fibres both with materials and spatial geometries of the fibre itself more and more researchers are studying the potential benefits of the various available fibres. One type of optical fibre which is showing promise and more recently with researchers taking a greater interest in is photonic crystal fibre (PCF) in conjunction with grating structures as fibre sensing devices; this is due to the "endless" geometric and material variations possible [2-5]. Particular interest is being shown in writing LPGs into PCF, whilst the sensitivity of LPGs is an advantage over fibre Bragg grating, this can cause problems of cross-sensitivity between parameters, with most common being temperature and another parameter which is of interest $[6,7]$. Some of the PCFs have shown very low temperature sensitivity thus giving a potential answer to the problem of cross-sensitivity/cross-talk between parameters being measured [8]. One of the major problems to date is the inscription of the LPG into the PCF due to the fact that the majority of PCF are made from pure-fused silica which is not photosensitive to UV laser radiation unless it is in the deep UV, thus the majority of traditional UV inscription techniques are not applicable. Several other inscription approaches have been used such as Electric-Arc method [8], $\mathrm{CO}_{2}$ lasers [9] and Femtosecond lasers [10] to overcome this problem with the Femtosecond laser technique being recognised as the most versatile.

*t.d.p.allsop@aston.ac.uk; kyriacos.kalli@.cut.ac.cy

Photonic Crystal Fibers III, edited by Kyriacos Kalli, Proc. of SPIE Vol. 7357

73570J - (C) 2009 SPIE · CCC code: 0277-786X/09/\$18 - doi: 10.1117/12.820790

Proc. of SPIE Vol. 7357 73570J-1 
Recent studies of LPGs written in standard telecoms fibre and PCF (specifically endless single mode (ESM) from Blaze Photonics) based upon a low repetition $(1 \mathrm{kHz}) 800 \mathrm{~nm}$ femtosecond laser by a point-by-point inscription scheme have shown post-fabrication spectrally instabilities at low temperatures $[11,12]$. Further to these studies we compare fusionarc to femtosecond fabricated LPGs the results show that at low temperatures (up to $200^{\circ} \mathrm{C}$ ) the fusion-arc LPG have greater stability. Also we show that at room temperature that the femtosecond laser LPGs exhibited both red and blue wavelength shifts with the magnitude of the shifts and that the time taken to achieve these maximum shifts are directly related to the inscribing power. Comparing these results to the fusion-arc LPGs suggests the thermal induced strain is a significant contribution to the overall index modification of the femtosecond LPGs and thus affects their overall spectral response.

\section{FABRICATION OF LONG PERIOD GRATINGS}

A series of LPGs with the same period $400 \mu \mathrm{m}$ were fabricated several with various inscription energies per pulse ranging from 410 to $650 \mathrm{~nJ}$ resulting in various grating lengths to obtain maximum strength attenuation bands. The alignment procedure used to inscribe the LPG into the central region of PCF (Blaze Photonics, ESM-1550-01) is done by visual inspection of the fibre from a camera system (see figure 1a) and given the specification of that fibre great care is taken to ensure that fibre is not twisted. A pre-inscription test is used to ensure that the LPG is written in the core, away from the inscription region of the fibre, test femtosecond laser "shots" are inscribed whilst the fibre is illuminated with green light, if the "shots" are in the central core region the green light is strongly scattered. The fusion-arc LPGs in the PCF are fabricated with a period of $400 \mu \mathrm{m}$ by the approach given in reference 8 .

The refractive index changes to create the LPG are introduced by a NIR femtosecond laser (800nm Spitfire/Evolution, Titanium/Sapphire system, Spectra-Physics Lasers). The femtosecond laser radiation was focused at a predetermined point near the core with a $\times 100(\mathrm{NA}=0.55)$ microscope objective, while the fibre was moved in a direction parallel to the fibre axis. The translation speed was $10 \mu \mathrm{ms}^{-1}$. A cylindrical lens $(\mathrm{f}=100 \mathrm{~mm})$ was put in close proximity to the microscope objective to shape the laser beam at the point of inscription. The femtosecond laser system produced a $1 \mathrm{kHz}$ train of $150 \mathrm{fs}$ pulses at $800 \mathrm{~nm}$, see Figure 1, other details of the experimental set-up can be found elsewhere [13].

The LPG growth characteristics were monitored using a broadband light source and optical spectrum analyser throughout the femtosecond laser inscription. Due to previous investigations into the spectral characteristics of femtosecond laser inscribed LPGs [13] the illuminating light used during fabrication was polarised, please see figure 1b. The polarisation of the light was constantly optimised to observe the growth of the LPGs attenuation bands during fabrication, and a polarisation controller was used to change the polarisation of the light illuminating the LPGs. Two examples of the transmission spectra are shown in figure $2 \mathrm{~b}$ and $2 \mathrm{c}$. 

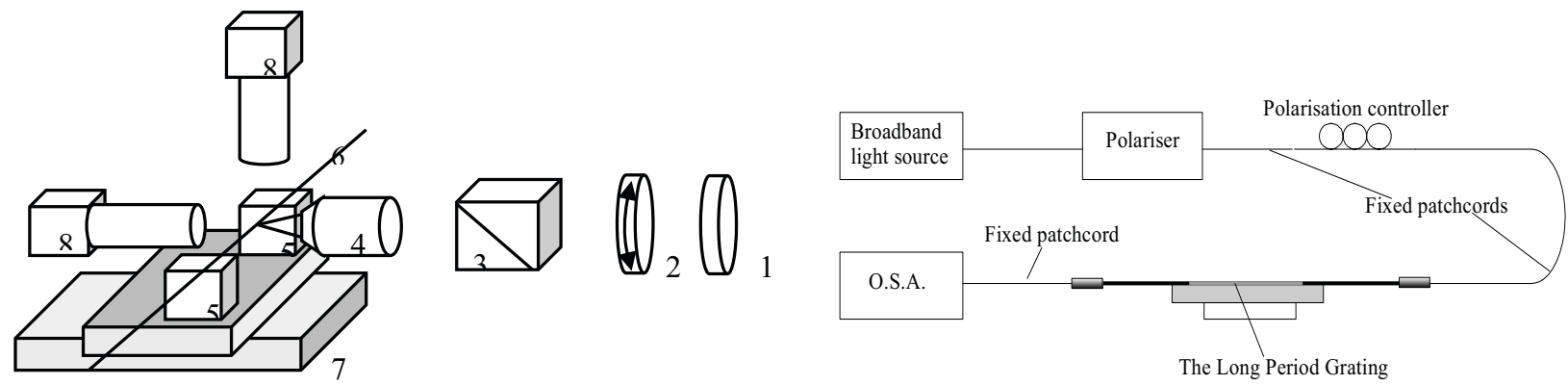

Figure 1 (a). Optical layout of inscription scheme. The light passes a shutter (1), a half-wave plate (2), Glan prism (3), x100 long working distance microscopic objective (4), and being focused in the fibre core (6). Two alignment 3D translation stages mounted on top of the high precision computer controlled 2D-stage (7). We use two CCD-cameras (8) with optical zooming system for alignment and on-line monitoring of the inscription process. (b) A schematic of the apparatus used to investigate the transmission spectra of the LPGs during fabrication
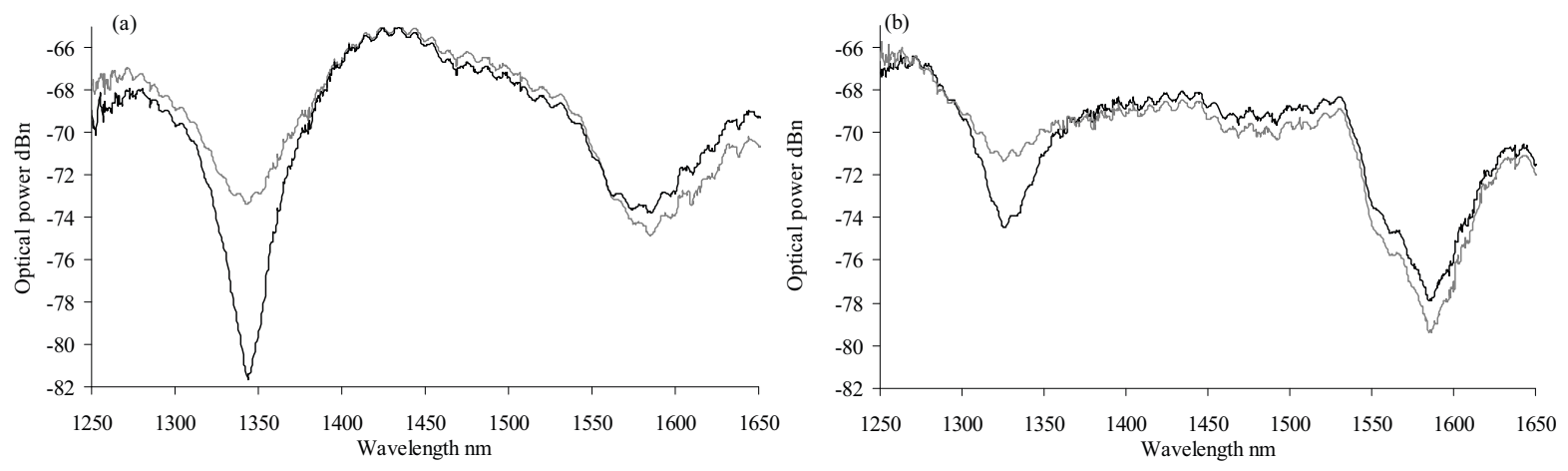

Figure 2 Typical examples of polarisation dependence on the transmission spectra of the femtosecond laser inscribed LPGs in PFC (ESM), (a) period of $400 \mu \mathrm{m}$, inscription energy $410 \mathrm{~nJ}$, length $9.6 \mathrm{~mm}$ (b) period of $400 \mu \mathrm{m}$, inscription energy $480 \mathrm{~nJ}$, length $10 \mathrm{~mm}$.

The polarisation dependence appears to have two main contributions one relating the symmetry of the inscription (other published work) and two the energy per pulse during inscription. The birefringence ranged from $10.2 \mathrm{~nm}$ to $1.4 \mathrm{~nm}$ over the energy range that was used.

\section{ANNEALING STUDIES}

\section{Annealing studies at room temperature}

The work thus far on the annealing studies have taken place at room temperature, $100^{\circ} \mathrm{C}$ and $200^{\circ} \mathrm{C}$, these temperatures were selected due to the fact these temperatures are used for the annealing of gratings in standard SMF fibre. The experimental arrangement is shown in figure $1 \mathrm{~b}$ for measurements taken at room temperature; at $100^{\circ} \mathrm{C}$ and $200^{\circ} \mathrm{C}$ the LPGs were placed into either an insulated box with a Peltier cooler or into temperature controlled furnace (a carbonlite tube furnace). At room temperature it was observed that LPGs displayed a post-fabrication spectral evolution. For all observed LPG the general trends appear to be the same with an initial red wavelength shift followed by a further blue wavelength shift, an example is shown in figure 3. 


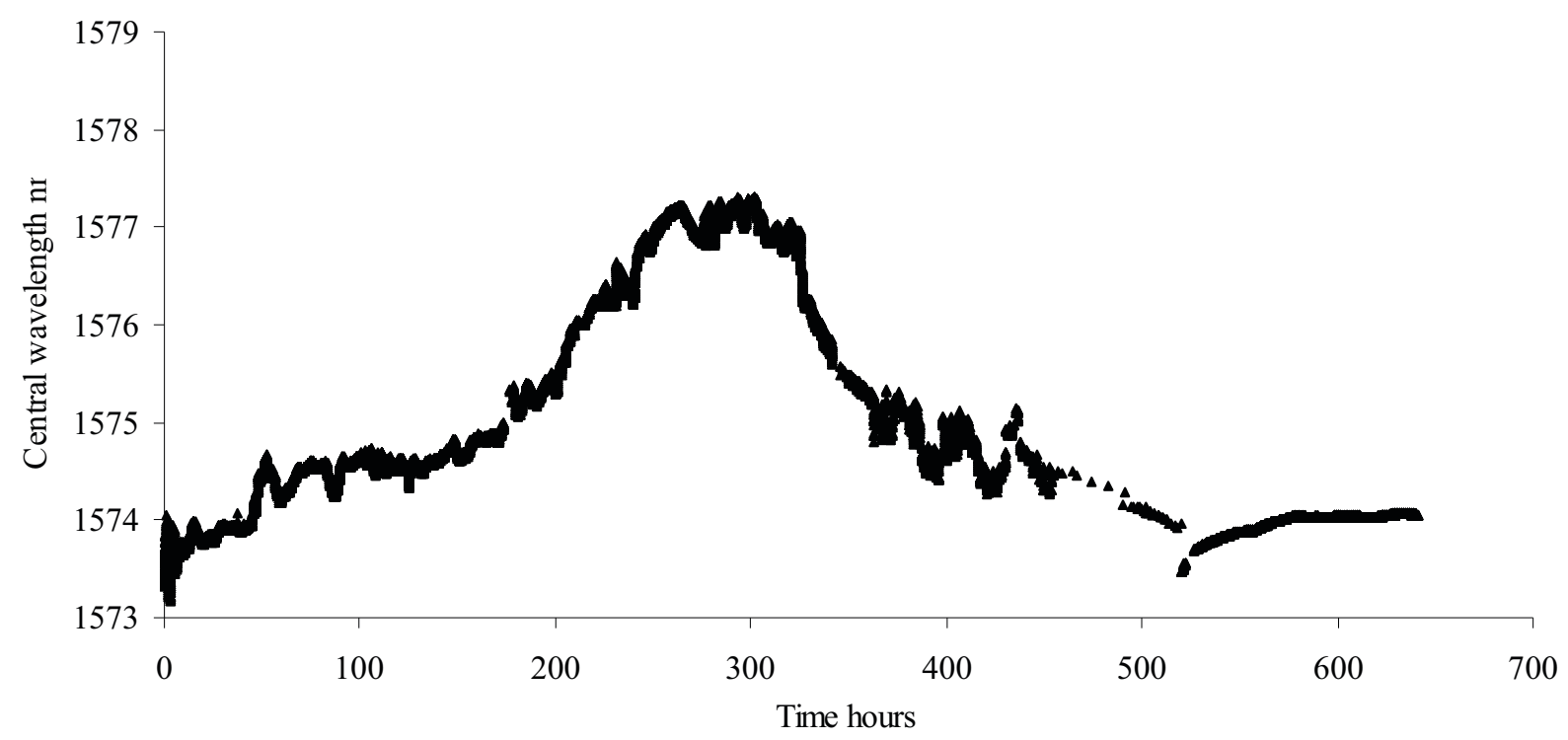

Figure 3 An example of the post fabrication spectral evolution of the peak wavelength of a femtosecond laser inscribed LPG in PCF (ESM) period $400 \mu \mathrm{m}$, energy $590 \mathrm{~nJ}$, length $7 \mathrm{~mm}$.

The largest spectral shifts appeared occurred in with the first 120 hours of fabrication with the maximum observed wavelengths were for the LPGs inscribed with the lowest powers, a red wavelength shift of $27.3 \mathrm{~nm}$ and the smallest shift occurring with the highest inscription energy; 650nJ producing $2 \mathrm{~nm}$ red shift. An overall trend of the wavelength shift for these LPG can be seen in figure 4. Also it appears that after approximately 800 hours the wavelength shifts become negligible.

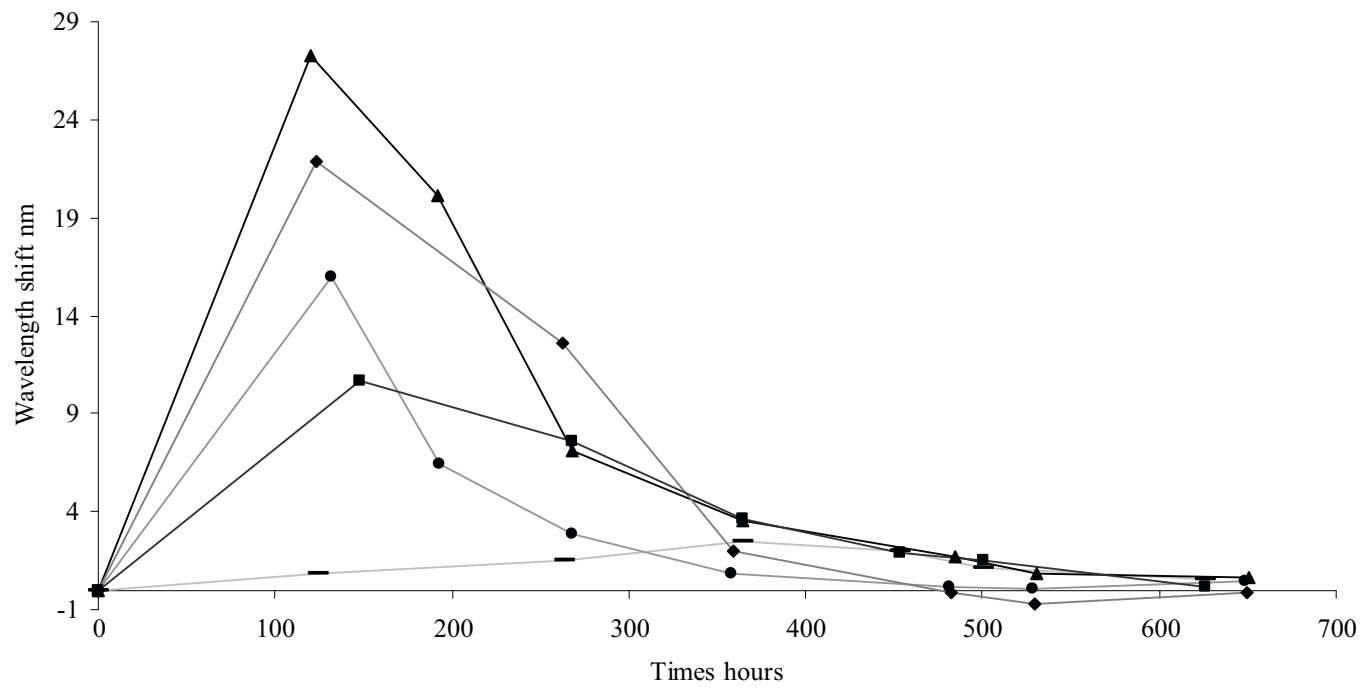

Figure 4 The observed wavelength shifts of the femtosecond laser LPGs in PCF (ESM) at room temperature energies used to inscribe the LPGs are $(\boldsymbol{\Delta}) 410 \mathrm{~nJ} /$ pulse, $(\bullet) 510 \mathrm{~nJ} /$ pulse, $(\bullet) 550 \mathrm{~nJ} / \mathrm{pulse},(\boldsymbol{\bullet}) 560 \mathrm{~nJ} / \mathrm{pulse},(\boldsymbol{)}) 650 \mathrm{~nJ} / \mathrm{pulse}$

It was found that at inscription energies below approximately $550 \mathrm{~nJ}$ produced a resultant blue shift and above produced red wavelength shifts, see figure 5a. Also the time taken to achieve the maximum appears to be related to the inscription energy, see figure $5 b$. 
(a)

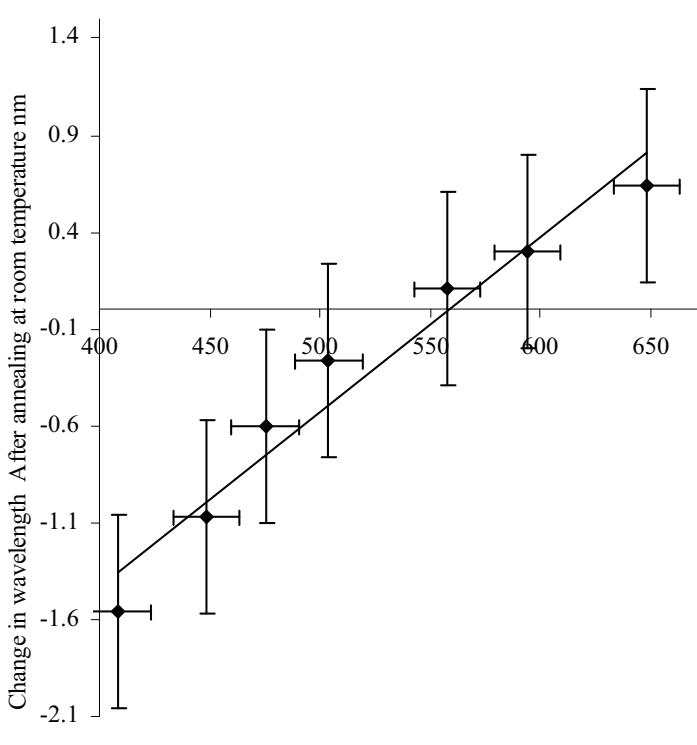

Inscription energy period nJoules

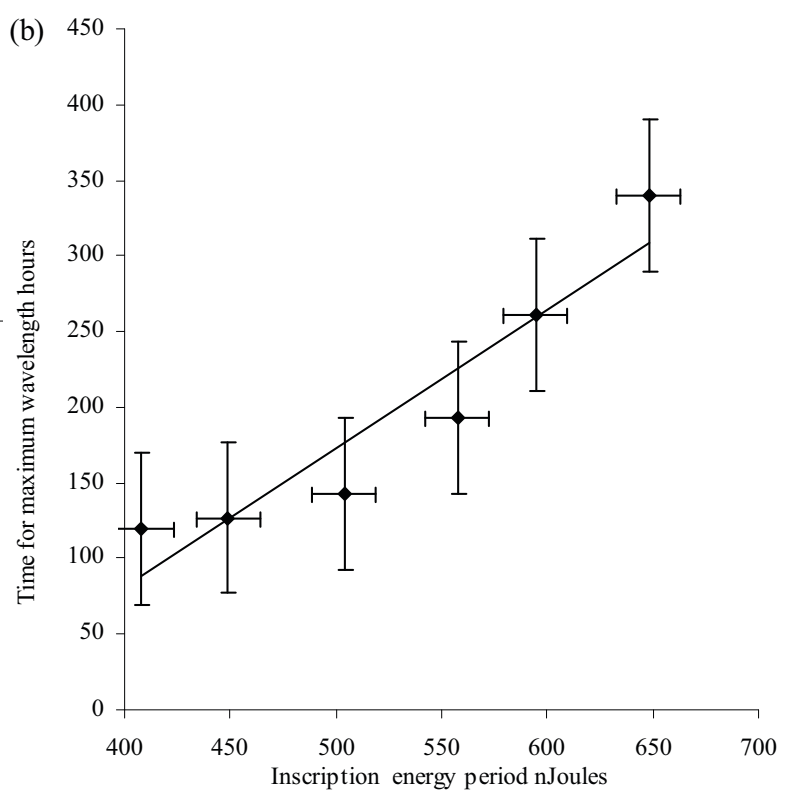

Figure 5 (a) The resultant wavelength shift after annealing at room temperature (b) the time of maximum observed wavelength shift

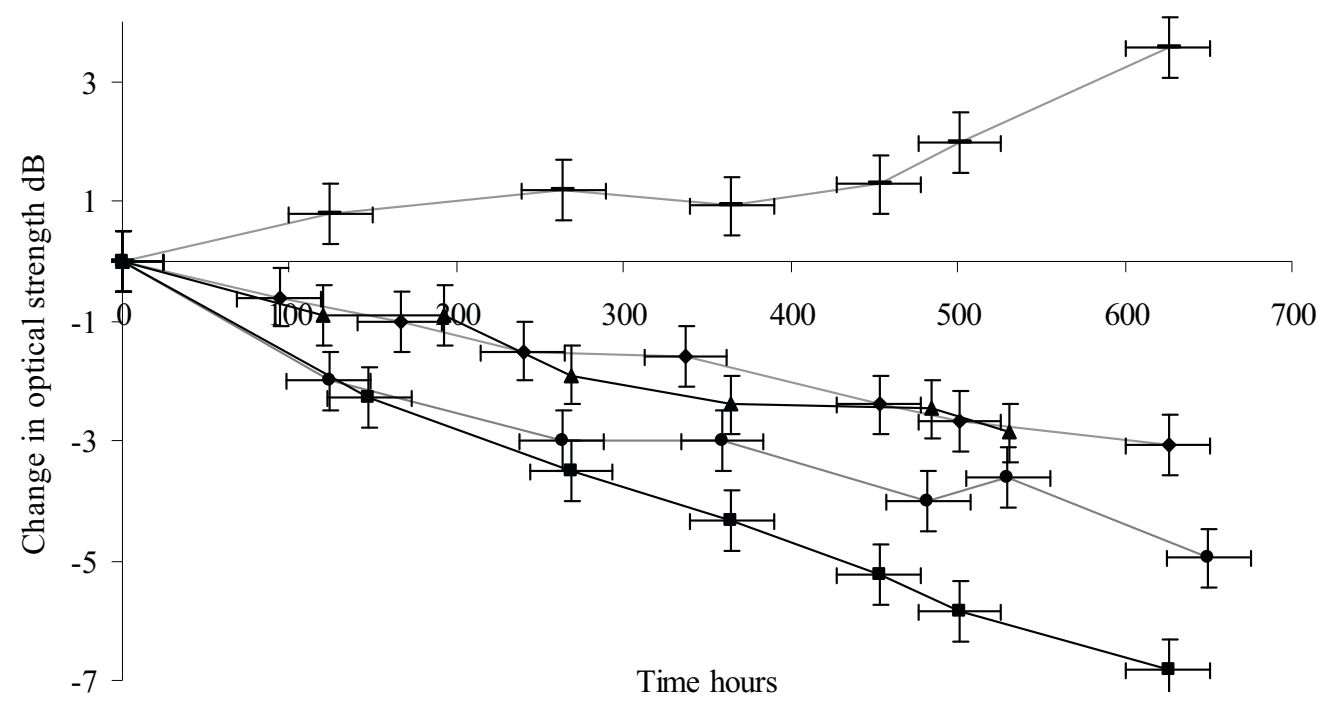

Figure 6 The observed change in the coupling strength of the femtosecond laser LPGs in PCF (ESM) at room temperature energies used to inscribe the LPGs are (A) $410 \mathrm{~nJ} /$ pulse, $(\bullet) 510 \mathrm{~nJ} / \mathrm{pulse},(\bullet) 550 \mathrm{~nJ} / \mathrm{pulse},(\bullet) 560 \mathrm{~nJ} / \mathrm{pulse},(-) 650 \mathrm{~nJ} / \mathrm{pulse}$

Figure 6 shows the change in optical strength of the LPGs with time. Comparing the results obtained for the femtosecond laser LPGs at room temperature to the fusion-arc LPGs, the fusion arc showed no wavelength shift or change in optical strength at room temperature. The fusion-arc LPGs results are not surprising due to other researchers published results in PCF and SMF fibres [8, 14] which show spectral stable LPGs up to temperatures of $600^{\circ} \mathrm{C}$. The published results for annealing experiments suggest that the main inscription mechanism for the index modification is compaction/densification. Annealing of the fusion-arc LPG at $100^{\circ} \mathrm{C}$ and $200^{\circ} \mathrm{C}$ yielded small variations compared with 
the femtosecond inscribed LPGs, examples of fusion arc LPG being annealed see figure 7 and figure 8 and femtosecond laser LPG is shown in figure 9 and 10.
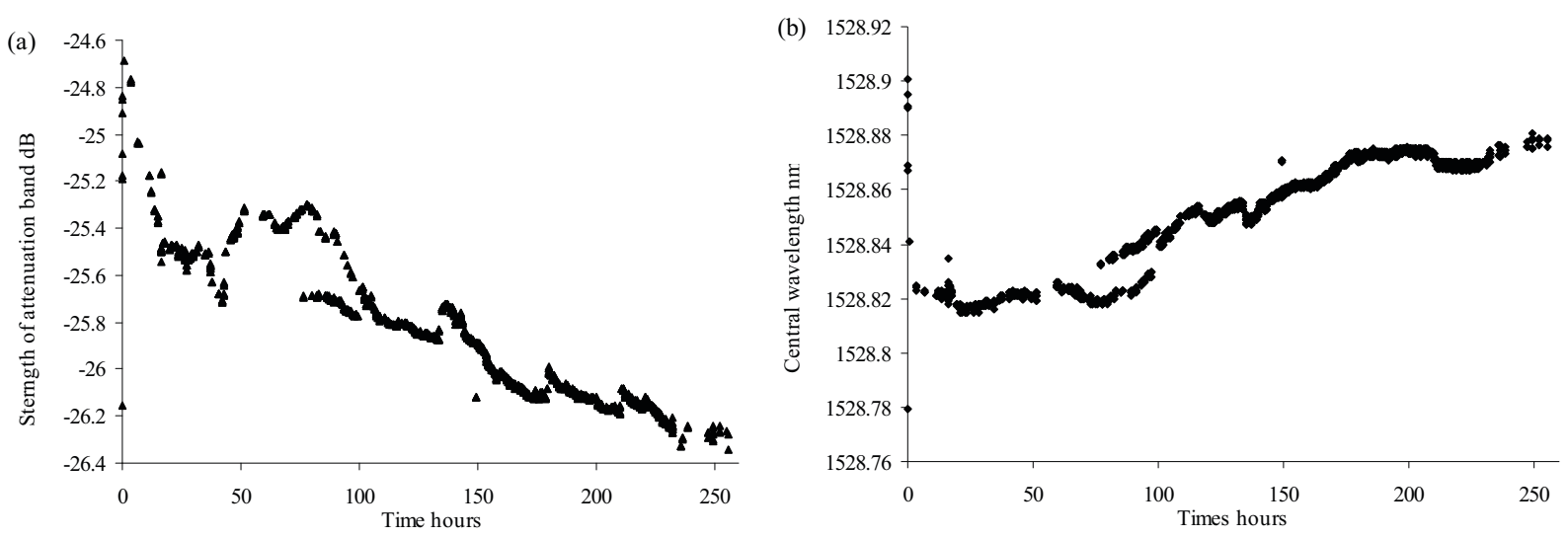

Figure 7 Example of the spectral variation of a fusion-arc fabricated LPG (period 400 $\mu \mathrm{m}$, length 20mm) in PCF (ESM) as a function of time being annealed at a temperature of $100^{\circ} \mathrm{C}$ (a) optical strength and (b) the central wavelength of attenuation band.
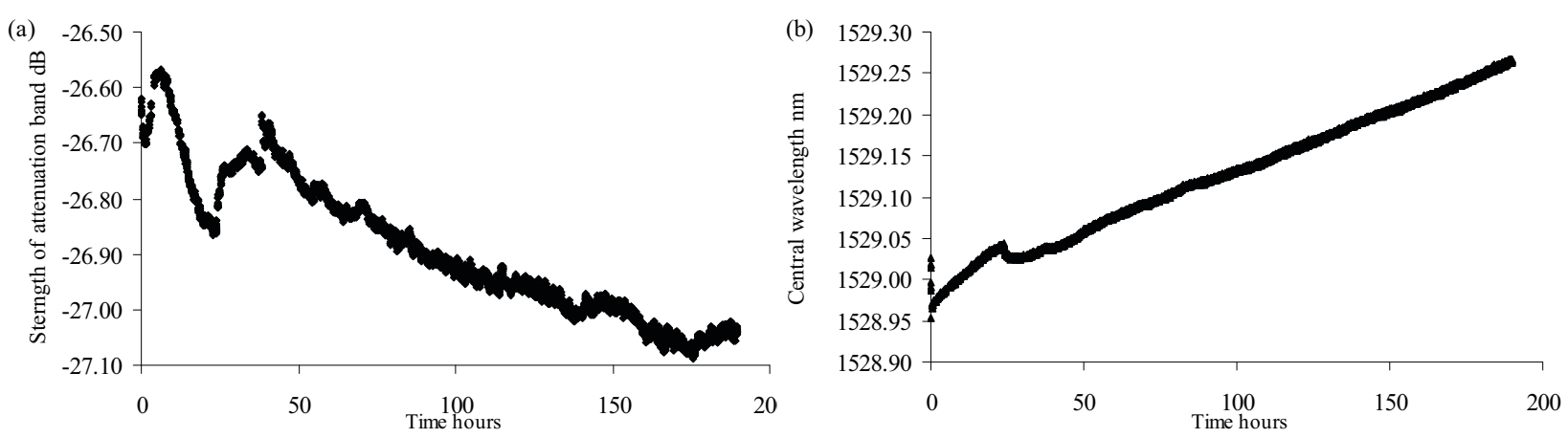

Figure 8 Example of the spectral variation of a fusion-arc fabricated LPG (period $400 \square \mathrm{m}$, length 20mm) in PCF (ESM) as a function of time being annealed at a temperature of $200^{\circ} \mathrm{C}$ (a) optical strength and (b) the central wavelength of attenuation band
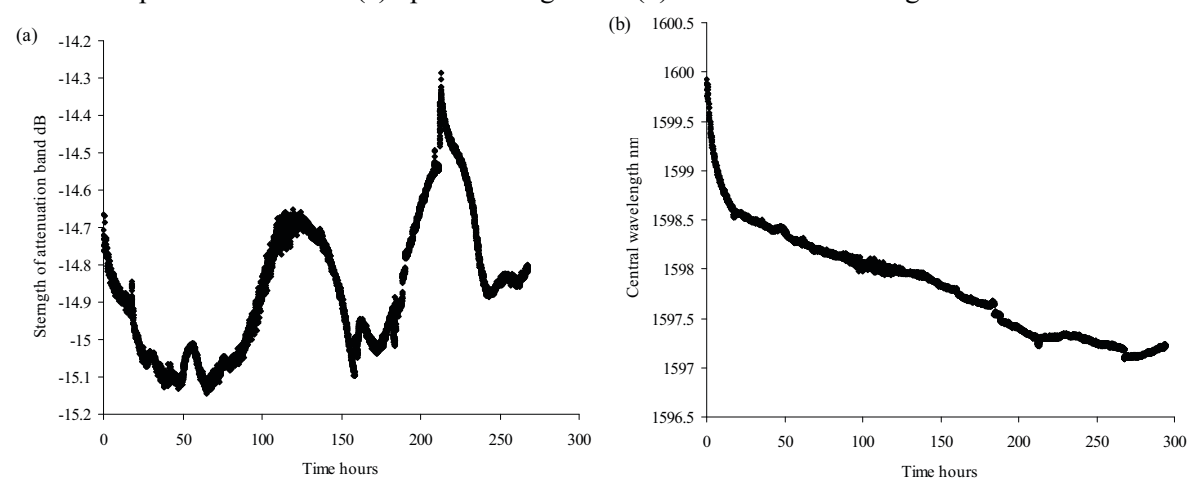

Figure 9 Example of the spectral variation of a femtosecond laser fabricated LPG (period $400 \mu \mathrm{m}$, length $9.6 \mathrm{~mm}$ inscription energy $410 \mathrm{~nJ}$ ) in PCF (ESM) as a function of time being annealed at a temperature of $100^{\circ} \mathrm{C}$ (a) optical strength and (b) the central wavelength of attenuation band. 

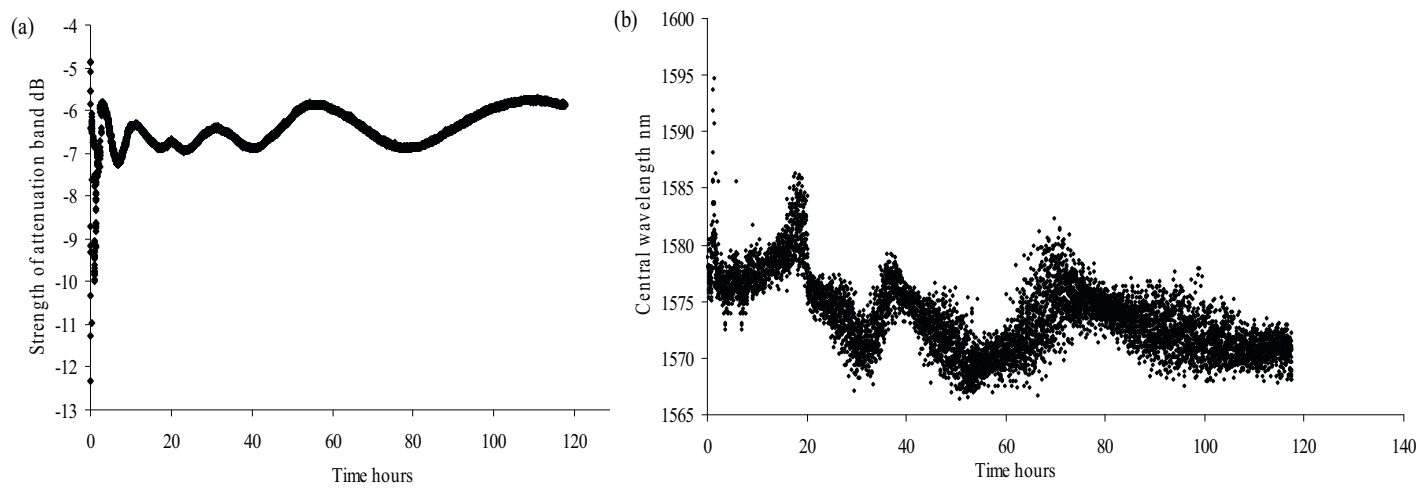

Figure 10 Example of the spectral variation of a femtosecond laser fabricated LPG (period $400 \mu \mathrm{m}$, length 9.6mm inscription energy $410 \mathrm{~nJ}$ ) in PCF (ESM) as a function of time being annealed at a temperature of $200^{\circ} \mathrm{C}$ (a) optical strength and (b) the central wavelength of attenuation band.

Comparing the fusion-arc LPG to the femtosecond laser inscribed LPG at annealing temperatures of room temperature $\left(25^{\circ} \mathrm{C}\right), 100^{\circ} \mathrm{C}$ and $200^{\circ} \mathrm{C}$ there appears to be significantly less post fabrication evolution of the fusion-arc LPG, with reference to figure 11. Also the higher the inscription energy for the femtosecond laser LPG the greater is the observed wavelength shift, see figure 11 .
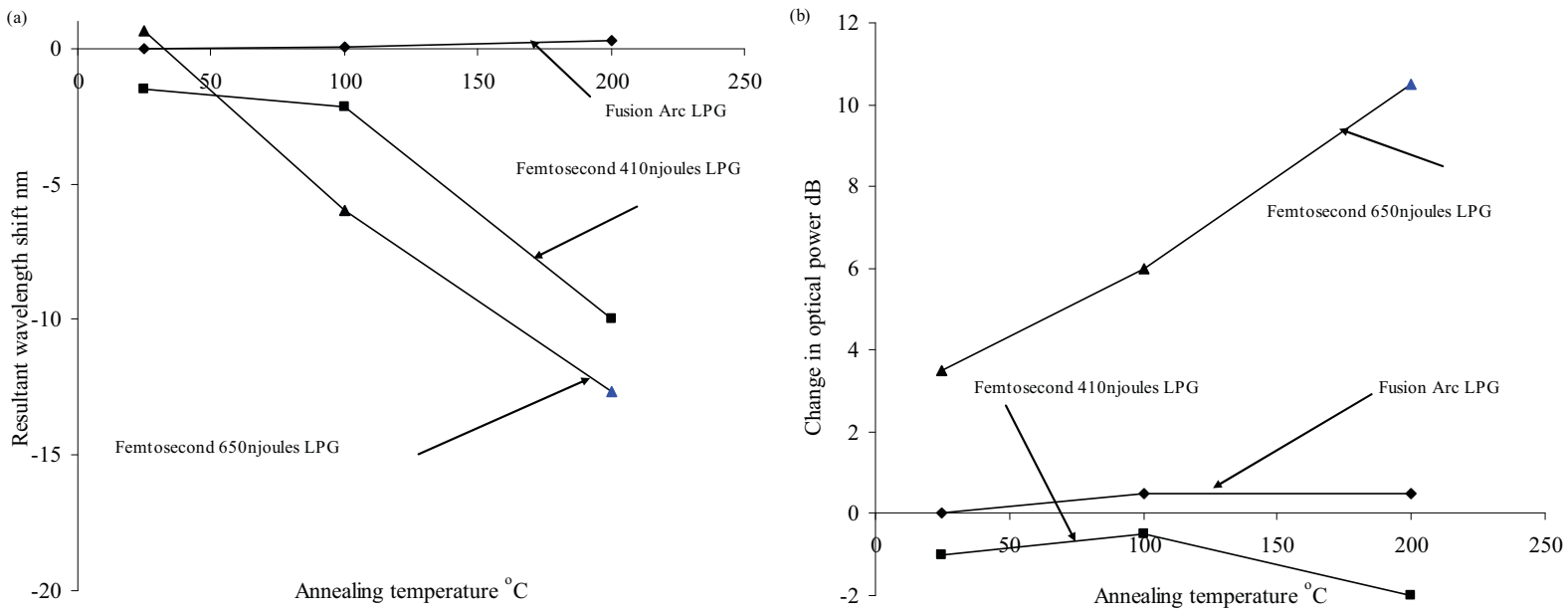

Figure 11 The observed wavelength shifts of LPGs in PCF (ESM) as a function of annealing temperature (a) wavelength shift (b) change in the optical strength.

\section{DISCUSSION}

The mechanisms responsible for the change of the index of refraction induced by the femtosecond laser pulses can vary, due to different laser parameters, such as energy, spot size, and the material properties. There are two effects that principally define the behaviour of the long period gratings at elevated temperature, which result from refractive index contributions that are either positive or negative. For low pulse energies refractive index profiles that are smooth and 
positive can readily be inscribed in planar silica samples, and these occur at similar values to the low pulse energies used here. We observe that the process of inscribing gratings using the femtosecond laser introduces a large degree of local birefringence that principally originates from the presence of residual stress, which results from lateral dimensional shrinkage of the material and localised densification. This supports the data that indicates for low pulse energies the material undergoes densification followed by a slow relaxation, even at room temperature, and for which heating accelerates this process of reaching a new equilibrium. This densification is not uniform but occurs in the direction of the laser focus into the optical fibre. We know that the femtosecond laser inscription process in general minimises the transport of heat to the surrounding material and that any cooling that takes place in the silica does so rapidly, with the passage of the laser beam. This means that there cannot be any long range reordering of the glass structure and that changes take place only at the location of the laser beam. When femtosecond laser pulses are focused inside a glass sample, the temperature of the irradiated region increases rapidly and the highest temperature is achieved at the centre of focus. As the temperature increases this leads to a conversion of the thermal expansion into plastic compressive strain; any free expansion of the heated zone is restricted by the surrounding cooler material. When the irradiated volume cools down, shrinkage of the heated material occurs. The thermal strain is totally cancelled out after the sample cools down, but not the compressive strain generated during the laser-induced heating. This residual compressive strain is responsible for local densification and consequently the increase of the refractive index of the glass. This highly local inscription process means that the fibre is comprised of regions that are laser-modified and completely unmodified. The principal negative index contribution arises from the creation of micro voids. In this case the relative index difference between the void and the surrounding fibre medium far outweighs the index change that results from densification. The magnitude of the index change of the void is at least one order of magnitude greater than that of the densification zone, which does contribute to the overall index change but on a smaller scale.

Inspecting the transmission data for annealing at room temperature for femtosecond inscribed LPGs shows that there is correlation between inscription energy and the resultant wavelength shift experience by the LPG, see figure 5a. The lower inscription energies produced net blue wavelength shifts, the largest blue shift being with the lowest energy (410nJoules) and the largest net red wavelength shift associated with the highest inscription energy. At room temperature all the femtosecond inscribed LPGs produced both red and blue wavelength shifts during their annealing time, see figure 3 and 4, whilst the fusion-arc LPGs showed negligible changes in both wavelength and optical strength. Annealing at the LPGs at higher temperatures $\left(100^{\circ} \mathrm{C}\right.$ and $\left.200^{\circ} \mathrm{C}\right)$ the high inscription energy (650nJoules) femotsecond LPG produced the greatest wavelength shift, see figure 11a but actually increased in optical strength compared to the other femtosecond LPGs, see figure $11 \mathrm{~b}$.

We observe throughout our data that there are particular laser energies for which we observe large LPG wavelength shifts, after which and over extended time periods, the samples reach equilibrium. The time to reach equilibrium at room and higher temperatures is shown to be laser energy dependent. At room temperature we observe that there is a threshold energy for which wavelength shifts occur over a constant time, after which higher laser energies are associated with far longer equilibrium times. In the latter cases this occurs as the degree of compressive strain has been locked in to the material at the higher pulse energy. Many measurements are clearly polarisation dependent and we observe changes that occur with femtosecond laser induced structures that are not observed in the case of the arc-induced LPG. This difference results from the almost completely circularly symmetric effect of the arc, which modifies the fibre properties without any preferential axis of inscription.

Considering the LPG theory [14] it is possible to obtain an estimate for the fraction/percentage of the index modification which is contributed by net thermal induced strain. It is know that at resonance (with the detuning parameter is zero) the transmission coefficient of a LPG can be expressed as $T=\cos ^{2}(\kappa \cdot L)$ where $L$ is the length of the grating and $\kappa$ is the coupling constant [14]. The coupling constant is given $\kappa=\frac{\omega \varepsilon_{0}}{4} \int_{0}^{2 \pi} d \phi \int_{0}^{\infty} r d r E_{1}^{*} \Delta n E_{2}$ where $E_{1}$ and $E_{2}$ are the transverse field distributions of the core and cladding mode and $\Delta \mathrm{n}$ is the peak change in the index modification. We assume that the index modification is radially symmetric and uniform (constant) across the core region of the fibre (the authors realise that this is not a realistic assumption due to the fact that LPG have polarisation dependence and due to the published literature on the subject of point-by-point femtosecond laser inscription technique) and that the E-field distributions of the core and cladding modes are unchanged over the annealing time period. Therefore, taking the ratio of 
the inverse cosine squared of the transmission coefficient at time $t$ of annealing to its initial inverse cosine squared of the transmission coefficient at time $t=0$ of annealing, i.e. $\frac{\cos ^{-2}(T(t))}{\cos ^{-2}(T(0))} \approx \frac{\Delta n(t)}{\Delta n(0)}$ will yield an estimate of the strain contribution to index modification, see table 1.

Table 1 Estimate of percentage of thermal induced strain to the overall index modification of the LPG

\begin{tabular}{|c|c|c|c|}
\hline Annealing Temperature & 25 & 100 & 200 \\
410 nJoules & -16.2 & -1.1 & -6.2 \\
& & 10.9 & 12 \\
650 nJoules & 2.6 & 0.08 & 0.025 \\
\hline
\end{tabular}

A general observation from the fabricated LPGs in the PCF is that fusion-arc LPGs have greater spectral stability at the three temperatures used for annealing than the femtosecond laser LPGs. It is known that the main index modification mechanism in fusion-arc LPGs is the compaction/densification of the glass $[15,16,17]$ and these grating structures are spectrally stable up to temperatures of approximately $600^{\circ} \mathrm{C}$ [15] whilst exhibiting low polarisation dependence. The fusion-arc LPGs showed negligible spectral changes, suggesting that the femtosecond inscribed LPG have a significant amount of thermal induced strain created during their fabrication, see Table 1 . We are undertaking further studies using a high repetition rate femtosecond laser systems to examine how LPGs inscribed under those conditions behave at room and high temperatures.

\section{REFERENCES}

[1] T. Allsop, D. Webb, I. Bennion," A comparison and characteristics of sensing parameters of long period gratings written in three different types of fiber", Optical Fiber Technology Vol. 9, Issue 4, pp 210-223, 2003

[2] K. Morishita, Y. Miyake, "Fabrication and Resonance Wavelengths of Long-Period Gratings Written in a Pure-Silica Photonic Crystal Fiber by the Glass Structure Change", J Lightwave Tech., Vol. 22, No. 2, pp.625-630, 2004.

[3] B. J. Eggleton, P. S. Westbrook, R. S. Windeler, S. Spälter, and T. A. Strasser, "Grating resonances in air-silica microstructured optical fibers", Optics Letts, Vol. 24, Issue 21, pp. 1460-1462, 1999.

[4] Y. L. Hoo, W. Jin, C. Shi, H. L. Ho, D. N. Wang, and S. C. Ruan, "Design and modeling of a photonic crystal fiber gas sensor," Appl. Opt. Vol. 42, pp.3509-3515, 2003.

[5] C. M. B. Cordeiro, M. A. R. Franco, G. Chesini, E. C. S. Barretto, R. Lwin, C. H. B. Cruz, and M. C. J. Large, “ Microstructuredcore optical fibre for evanescent sensing applications" Optics Express, Vol. 14, Issue 26, pp. 13056-13066, 2006

[6] Y. Wang, L. Xiao, D. N. Wang, and W. Jin, "In-fiber polarizer based on a long-period fiber grating written on photonic crystal fiber", Optics Letts., Vol. 32, No. 9, pp. 1035-1037, 2007.

[7] Z. He, Y. Zhu, and H. Du, "Long-period gratings inscribed in air- and water-filled photonic crystal fiber for refractometric sensing of aqueous solution", Appl. Phys. Lett., Vol. 92, Is. 4, pp. 044105. 2008

[8] H. Dobb, K. Kalli, D.J. Webb, "Temperature-insensitive long period grating sensors in photonic crystal fibre”, Vol.40, Is. 11, pp.657- 658, 2004.

[9]Y. Zhu, P. Shum, J-H Chong, M. K. Rao, and C. Lu, "Deep-notch, ultracompact long-period grating in a large-mode-area photonic crystal fiber", Optics Letts., Vol. 28, No. 24, pp.2467-2469, 2003.

[10] G. Brambilla, A. A. Fotiadi, S. A. Slattery and D. N. Nikogosyan, "Two-photon photochemical long-period grating fabrication in pure-fused-silica photonic crystal fiber," Optics Letts., Vol. 31, No. 18, pp.2675-2677, 2006.

[11] T. Allsop, K. Kalli, K. Zhou, G. Smith, M. Dubov, K. Sugden, D. Webb, I. Bennion, "Annealing and spectral characteristics of femtosecond laser inscribed long-period gratings written into a photonic crystal fibre" OFS-19, Perth, West Australia, 2008

[12] T. Allsop, K. Kalli, K. Zhou, G. Smith, Y. Lai, M. Dubov, K. Sugden, D. Webb, I. Bennion, M. Komodromos, "Characterisation of femtosecond laser inscribed long period gratings in photonic crystal fibre", Proceedings of Photonic Crystal Fibers II, SPIE Vol. 6990, pp.264, 2008

[13] A. Martinez, "Point by point FBG inscription by a focused NIR femtosecond laser", OSA Conf.Proc. CLEO/IQEC and PhAST Washington, DC, 2004, CMY6

[14] P. Palai, M. Das, B.P. Pal, "Characterization and simulation of long period gratings fabricated using electric discharge", Vol.193, pp.181-185, 2001. 
[15] G. Humbert, A. Malki, "Characterizations at very high temperature of electric arc-induced long-period fiber gratings", Optics Comms., Vol. 208, pp. 329-335, 2002.

[16] K. Morishita and A, Kaino, "Adjusting resonance wavelengths of long-period fiber gratings by the glass-structure change", Applied Optics, Vol.44, No. 24, pp.5018-5023, 2005

[17] T. Allsop, M. Dubov, H. Dobb, A. Main, A. Martinez, K. Kalli, D. J. Webb, and I. Bennion, “A comparison of the spectral properties of high temperature annealed long-period gratings inscribed by fs laser, UV, and fusion-arc", Reliability of Optical Fiber Components, Devices, Systems, and Networks III, SPIE Vol 6193, Munich, Germany, 2006. 